\title{
Nordiques
}

39 | 2020

Varia

\section{Enseigner les verbes de position suédois avec l'aide de la sémantique cognitive}

\section{Maria Hellerstedt}

\section{(2) OpenEdition}

1 Journals

Édition électronique

URL : http://journals.openedition.org/nordiques/575

DOI : $10.4000 /$ nordiques.575

ISSN : $2777-8479$

Éditeur :

Association Norden, Bibliothèque de Caen la mer

Référence électronique

Maria Hellerstedt, «Enseigner les verbes de position suédois avec l'aide de la sémantique cognitive », Nordiques [En ligne], 39 | 2020, mis en ligne le 01 novembre 2020, consulté le 13 mars 2021. URL: http://journals.openedition.org/nordiques/575; DOI : https://doi.org/10.4000/nordiques.575

Ce document a été généré automatiquement le 13 mars 2021

Nordiques 


\title{
Enseigner les verbes de position suédois avec l'aide de la sémantique cognitive
}

\author{
Maria Hellerstedt
}

\section{Introduction}

1 Dans cet article, nous présenterons une étude exploratoire concernant l'évolution d'apprentissage de certains verbes suédois par des apprenants francophones avant et après un enseignement ciblé et précis. Il s'agit des verbes dit de position statiques (ligga " être couché ", sitta « être assis », stå « être debout »1). Ces verbes ne décrivent pas uniquement la posture de l'être humain, mais aussi la localisation statique de quelqu'un ou de quelque chose à un endroit donné. Par exemple, la phrase « le téléphone portable est posé sur l'étagère » sera rendue en suédois par mobiltelefonen ligger på hyllan. Alors qu'en français, la copule "être» est utilisée, le suédois implique que le locuteur choisisse parmi les verbes de position statiques afin d'encoder cette situation. En l'occurrence, le verbe ligga "être couché » sera le seul verbe de position possible, et nous allons en exposer les raisons ci-dessous. Cependant, il existe également en suédois l'équivalent du verbe "être ", vara, qui peut être considéré comme un hypéronyme, décrivant la localisation concrète d'un être humain ou d'un objet. Dans une étude antérieure ${ }^{2}$, nous avons pu constater que la situation d'usage de ce type de verbe n'est pas identique à celle d'un verbe de position et que parfois, l'usage du verbe positionellement neutre vara manque d'idiomaticité et peut être vu comme une interférence du français. Les locuteurs natifs du suédois semblent préférer un verbe de position à un verbe neutre pour localiser un objet : la position est encodée dans 55,3\% des cas.

2 Le locuteur francophone, qui a recours à un verbe très général pour encoder la position du téléphone dans sa langue maternelle, aura des difficultés à choisir entre ces verbes suédois. Dans cet article, après avoir rendu compte des différences typologiques entre 
le suédois et le français, ainsi que de la sémantique des verbes, nous présenterons un état de l'art qui exposent ces difficultés et en expliquent les raisons. Ensuite, nous proposerons une nouvelle façon d'enseigner les verbes de position, et exposerons les résultats d'une étude pilote sur l'évolution des compétences des apprenants après un tel enseignement.

3 Les verbes de position ayant une sémantique complexe, même pour un apprenant de suédois langue maternelle ${ }^{3}$, nous posons l'hypothèse qu'avec un enseignement ciblé et très précis concernant les sens et les usages, basé sur la sémantique cognitive, les apprenants francophones adultes acquerront une meilleure compréhension de ces verbes.

\section{L'arrière-plan}

\section{Typologie}

Les verbes de position décrivent la localisation ainsi que la posture d'un être humain, d'un objet concret ou abstrait. Les traits sémantiques de ces verbes sont par conséquent objet + localisation + position. En suédois, toutes ces informations se trouvent lexicalisées dans le verbe. Les verbes correspondant en français, à savoir " être ", "se trouver ", "y avoir», excluent la composante position, car aucune précision sur l'orientation de l'objet localisé ne se trouve dans le verbe.

5 La relation spatiale entre la Figure (l'objet localisé) et le Fond (l'endroit où se trouve la Figure) est schématisée par les concepts décrits par des traits sémantiques. Selon Talmy ${ }^{4}$, ceux-ci peuvent être exprimés linguistiquement lorsqu'on encode une situation d'emplacement, mais ne le sont pas obligatoirement. La position de l'objet, appelée la Manière par Talmy, est un composant sémantique présent en suédois, mais rarement en français. Ceci relève de deux modèles de lexicalisation différents, qui répartissent les deux langues en deux typologies, à savoir les langues à cadrage verbal, qui ne lexicalisent pas la Manière dans le verbe, et les langues à satellite, où la sémantique du verbe de localisation inclut souvent la Manière, exprimée par la position. Ainsi, le suédois est une langue à satellites tandis que le français est une langue à cadrage verbal.

Baron et Herslund ${ }^{5}$ l'illustrent un peu différemment, mais le principe est le même. Selon ces deux chercheurs, les langues dites endocentriques, comme le danois et le suédois, encodent le poids lexical au centre de la proposition, à savoir dans le verbe, tandis que dans les langues dites exocentriques, comme le français, les verbes n'ont qu'un sens général ${ }^{6}$. Herslund ${ }^{7}$ explique ce phénomène par une concrétisation des événements chez les langues endocentriques, exprimée par la manière dans les verbes. Par exemple, les verbes danois skcere (" couper avec un couteau »), klippe (" couper avec des ciseaux »), save (" couper avec une scie »), hugge (" couper avec une hache») sont tous, la plupart du temps, traduits en français par «couper », ce qui rend le concept plus général. Ainsi, en français, moins d'informations sur l'entité qui subit l'activité sont données via le verbe, tandis que le verbe danois, tout comme le verbe suédois, pose des exigences sur la référence du nom en relation avec le verbe (sujet ou objet). Il semblerait donc que les verbes de position suédois peuvent être considérés comme des classificateurs de noms, comme proposé par Lemmens \& Perrez $^{8}$. Ces verbes classificateurs catégorisent les Figures, en posant des exigences sur leurs propriétés 
afin d'être combinés avec celles-ci. Nous développerons cet aspect sous la rubrique consacrée aux paramètres sémantiques des verbes.

\section{La sémantique des verbes de position}

7 Le sens prototypique des verbes de position indique la posture humaine. Il s'agit donc de leur sens positionnel. Mais les verbes de position statiques encodent également la localisation d'une personne ou d'un objet, concrètement ou métaphoriquement ${ }^{9}$. On parlera respectivement des sens spatiaux et métaphoriques. En français, pour l'aspect spatial, ils seront majoritairement rendus par les verbes "être», "y avoir» ou "se trouver ", comme mentionné précédemment. Ces trois verbes sont également utilisés en suédois dans une construction grammaticalisée exprimant l'aspect progressif ${ }^{10}$. Il n'est donc pas étonnant de constater que ces verbes font partie des verbes les plus fréquents dans la langue suédoise. En effet, sur une liste de fréquence des verbes suédois, établie par Viberg à l'aide du corpus Stockholm Umeå Corpus 1997'11, l'on trouve stå « être debout » en 17e, ligga " être couché » en 23e et sitta " être assis » en $35 \mathrm{e}$ position. En excluant les usages métaphoriques et grammaticalisés de ces verbes ${ }^{12}$, il semble que les verbes stå et ligga changent de place, surtout en raison de l'usage imposé du verbe ligga pour l'emplacement géotopographique, considéré comme un usage concret de localisation.

\section{Les paramètres sémantiques}

8 Les paramètres sémantiques des verbes de position sont élaborés à partir des représentations mentales et des schémas d'images d'un être humain et de ses postures ${ }^{13}$. En effet, au sein de la sémantique cognitive ${ }^{14}$, on considère que l'expérience corporelle est la base de la compréhension du monde qui nous entoure, et que celle-ci se reflète dans certaines expressions linguistiques. Pour les verbes de position, il est facile de voir le rôle que joue le corps dans leurs significations, et si les apprenants s'emparent de ces sensations, il est fort possible qu'ils les acquièrent moins laborieusement.

9 Les paramètres sémantiques du verbe stå « être debout » peuvent être synthétisés de la manière suivante ${ }^{15}$ :

- être sur ses pieds $\rightarrow$ être sur sa base

- avoir une extension vers le haut et partir de la base

- avoir une extension verticale

- être dans une position canonique

- être fonctionnel

Ainsi, une personne qui est debout se trouve sur ses pieds, ayant une extension verticale à partir de ces pieds. En outre, c'est dans cette position que nous nous attendons à voir un être humain, la posture pour ainsi dire standard appelée position canonique. En effet, le signifié d'un être humain sera une personne en position debout. C'est également dans cette posture que l'être humain est le plus fonctionnel, prêt à se mettre en action, à agir ou à se mettre en mouvement. On peut transférer ces observations à des objets concrets, par exemple à une bouteille comme dans la phrase suivante : Flaskan står på stolen « La bouteille est (debout) sur la chaise ». Cette bouteille repose sur sa base, a une extension verticale à partir de sa base et elle se trouve dans 
une position attendue et normale, c'est-à-dire canonique. On peut également considérer que la bouteille est fonctionnelle en tant que récipient lorsqu'elle est debout. C'est pourquoi, en suédois, il faut encoder cette situation par le verbe de position stå. Une Figure doit donc avoir une des propriétés énumérées ici, comme la base, la verticalité, la fonctionnalité ou la canonicité, pour être encodée par ce verbe. Ainsi, nous voyons cette interaction entre le sens du verbe et la catégorie de la Figure qui suscite la théorie sur les verbes en tant que classificateurs de noms.

11 Le verbe ligga « être couché, allongé » est en grande partie l'opposé sémantique de stå. Voici les paramètres sémantiques, applicables avant tout à un être humain en position allongée ${ }^{16}$ :

- être sur une de ses côtés

- ne pas être sur sa base (ses pieds)

- avoir une orientation horizontale

- être non fonctionnel (non-canonique pour les objets normalement encodés par stå)

- être un objet sans dimension/rond

12 En outre, ce verbe est celui qui encode les emplacements géotopographiques, par exemple Stockholm ligger $i$ Sverige "Stockholm se trouve en Suède ». Ces paramètres peuvent également être appliqués à une situation comprenant un objet concret, comme dans Pennan ligger på bordet " Le crayon est (couché) sur la table ", décrivant un crayon qui se trouve sur un de ses côtés avec une orientation horizontale. Il n'a pas de véritable base, et sa fonctionnalité n'est en cours que lorsqu'il est utilisé. Le paramètre de la canonicité semble être hors-jeu, comme il n'y a pas de position standard pour un crayon qui peut aussi bien être posé notamment sur une table que dans un pot de crayon. La phrase Bollen ligger på gräsmattan (Le ballon est (couché) sur la pelouse), illustre la contrainte d'utiliser le verbe ligga pour tous les objets ayant une extension symétrique, voire ronde, comme un ballon, un caillou ou un dé. Pour le dernier paramètre, tous les apprenants de suédois langue étrangère s'étonnent de voir, mais apprennent vite, qu'un endroit géotopographique est encodé par le verbe ligga, par exemple dans Stockholm ligger i Sverige «Stockholm se trouve (couché) en Suède ». Ainsi, la Figure encodée par le verbe ligga peut avoir une extension horizontale, ne pas avoir de base ou ne plus se trouver sur sa base, être non-fonctionnelle, voire non-canonique, sauf s'il s'agit d'un objet symétrique ou d'une relation géotopographique.

13 Le troisième verbe de position, sitta " être assis ", a des sens élargis qui paraissent loin du sens original, à savoir une personne assise. Les deux paramètres qui couvrent les utilisations élargies sont les suivants ${ }^{17}$ :

- « contact » - la Figure est en contact avec le Fond, voire attachée à lui

- «inclusion » - la Figure se trouve en partie insérée dans un Fond étroit, voire contenu par le Fond

Ces paramètres découlent également de la posture humaine et de l'image d'une personne assise en contact avec une chaise. En effet, la chaise ayant un siège et un dossier, l'on pourrait s'imaginer que celle-ci contient la personne. Ainsi, dans l'exemple Affischen sitter på väggen "L'affiche est (assis) sur le mur", c'est la proximité entre l'affiche et le mur qui est encodée par le verbe sitta. Pour les petits animaux, qui dans leur position naturelle sont en contact avec le fond, on utilise aussi ce verbe en suédois. Ainsi, un oiseau sur une branche, un lapin ou une souris par terre, une araignée par terre ou sur le mur, sont encodés par le verbe sitta, comme dans Fågeln sitter $i$ trädet «L'oiseau est (assis) dans l'arbre ». Les lapins et les souris ne peuvent être codés que 
par le verbe sitta lorsqu'ils se trouvent dans leur position normale. En effet, étant couchés, ils dorment ou ils sont morts, et étant debout, ils se dressent sur leurs pattes arrières. Une araignée, en revanche, ne peut être qu' «assise » et jamais « debout ». Si elle est couchée, elle est morte, même si elle n'a pas vraiment changé de position. L'accord entre le verbe sitta et la Figure qu'il encode semble donc se trouver dans le contact intime entre la Figure et le Fond ${ }^{18}$.

Afin de choisir entre ces verbes pour encoder une situation de localisation, il faut donc avoir une certaine connaissance de leur sémantique, mais aussi du monde et des objets qu'on y trouve. Ces derniers vont avoir une influence sur le choix du verbe de par leur nature ainsi que leur extension dans l'espace. Or, tous les paramètres ne sont pas toujours actualisés dans chaque situation spatiale, ce qui rend la tâche encore plus laborieuse pour l'apprenant.

\section{L'acquisition des verbes de position}

16 Il existe très peu d'études sur l'acquisition des verbes de position statiques suédois par des apprenants L2. La majorité des projets ${ }^{19}$ a porté sur les verbes de position de placement, c'est-à-dire les équivalents causatifs des verbes étudiés dans cet article, à savoir ställa « mettre debout », lägga « mettre couché, allonger » et sätta « mettre assis, asseoir ", qui en français sont traduits la plupart du temps par les verbes généraux de placement «mettre, poser, placer». Pour d'autres langues, il existe quelques études d'acquisition des verbes de placement, par exemple pour le néerlandais L2 appris par des anglophones ${ }^{20}$, ou le danois L2 appris par des hispanophones ${ }^{21}$. Les résultats les plus pertinents de ces études portent sur la tendance à apprendre les verbes de position statiques avant les verbes de position de placement et à utiliser, d'une façon erronée, les premiers à la place des seconds. En outre, il semble que le paramètre de l'horizontalité soit le plus transparent et le plus facile à apprendre pour les apprenants L2. Par conséquent, une certaine sur-utilisation de ce concept semble être de mise, même lorsqu'il n'est pas présent dans le contexte. Ainsi, on trouve dans toutes ces études une tendance à généraliser un des verbes de placement pour un grand nombre de contextes de placement. En outre, on y constate qu'il est particulièrement difficile pour l'apprenant de diviser une catégorie dans sa langue maternelle en deux catégories dans la L2, c'est-à-dire d'aller de un à plusieurs et du général au spécifique ${ }^{22}$. De la sorte, pour apprendre les verbes de position, les francophones doivent diviser les catégories de la localisation et du placement en plusieurs catégories plus spécifiques, qui prennent en compte l'orientation de la Figure ${ }^{23}$.

Les paramètres décrits ici sont surtout applicables aux usages spatiaux des verbes de position. Par conséquent, malgré la fréquence et l'utilité de ces verbes dans la vie quotidienne, il est fort probable qu'ils posent un grand problème aux locuteurs francophones apprenant le suédois L2. Ces problèmes reposent donc sur trois points ${ }^{24}$ :

1. la flexibilité de l'encodage de chaque verbe, c'est-à-dire la polysémie et les différentes situations où le verbe peut être appliqué (la variation sémasiologique)

2. la variation de l'encodage: une seule et même situation spatiale peut être encodée par plusieurs verbes (la variation onomasiologique)

3. la contrainte de l'encodage, le fait que les verbes de position sont obligatoires (ou presque) pour encoder les situations spatiales. 
18 localisation sont celles réalisées sur l'acquisition du néerlandais par les francophones belges ${ }^{25}$ et la nôtre ${ }^{26}$, faite sur l'apprentissage du suédois par les francophones. Les informateurs de l'étude de Lemmens et Perrez ont effectué une production écrite spontanée, tandis que dans notre étude, deux groupes d'apprenants (niveau intermédiaire et avancé) ont décrit à l'oral des images dans lesquelles il fallait localiser des personnes et des objets. Selon les résultats de Lemmens et Perrez, le verbe staan " être debout " est le plus employé par les apprenants L2, étant en même temps le verbe de position statique le plus fréquent en néerlandais ${ }^{27}$. Aussi la sur-utilisation du verbe staan est en concordance avec l'input que ces apprenants reçoivent, et ils appliquent donc les modèles dominants de la langue cible ${ }^{28}$. Ce constat met en question l'universalité du verbe exprimant l'horizontalité, proposée ci-dessus, et prend plutôt en compte l'importance de la fréquence. Son équivalent suédois, stå, est également le verbe de position le plus fréquemment utilisé selon la liste de fréquence (place 17). Or, dans notre étude, nous avons pu constater une sur-utilisation du verbe ligga, qui se trouve à la place 23 sur la liste de fréquence. Toutefois, ce verbe peut être considéré comme le verbe le moins lié à une relation spatiale précise, car il n'encode pas seulement l'emplacement des objets concrets, mais aussi les relations géotopographiques ${ }^{29}$. En ce qui concerne le verbe sitta, il semble être acquis en dernier lieu, selon nos résultats. Cela peut être dû à une plus grande facilité à percevoir les paramètres de la verticalité et de l'horizontalité respectivement, tandis que les paramètres du contact et de l'inclusion peuvent paraître plus abstraits et moins évidents ${ }^{30}$.

De façon générale, les verbes de position sont beaucoup utilisés par les locuteurs francophones apprenant le suédois, à savoir dans 54,8\% des cas pour les apprenants au niveau intermédiaire et dans $75,3 \%$ pour les apprenants avancés (à comparer avec les $79,2 \%$ des locuteurs natifs). Les autres stratégies utilisées incluent surtout l'usage d'un verbe positionnellement neutre (comme vara, finnas), ou d'une ellipse verbale ${ }^{31}$.

\section{La méthode}

L'étude pilote porte sur 13 étudiants francophones en 2e année de la licence de suédois à l'université de Lille. Grands débutants au départ, ils étudient le suédois depuis un an et demi. Par conséquent, au moment de l'étude, ils ont l'équivalent d'un niveau A2-B1 (selon le cadre européen commun de référence: $\mathrm{CECR}^{32}$ ). Dans leur parcours, ils ont bien évidemment déjà rencontré ces verbes, mais ce n'est qu'en deuxième année que ceux-ci sont abordés d'une façon plus systématique lors des cours de grammaire. Afin de pallier les difficultés d'usage des verbes de position, mises en évidence dans notre étude antérieure ${ }^{33}$, nous leur présentons ces verbes d'une façon très ciblée, en expliquant en détail les paramètres sémantiques exposés ci-dessus avec des exemples et des mises en situation, avant de leur faire faire un grand nombre d'exercices, notamment en décrivant des images. Afin d'établir les connaissances préalables des étudiants sur l'usage des verbes de position, un questionnaire à choix multiple leur est soumis avant l'explication détaillée. Le choix de ce type de questionnaire a été motivé pour sa facilité à être intégré dans l'enseignement, car cet exercice n'est pas très chronophage et les étudiants comprennent vite ce qu'il faut faire. Dans le questionnaire, il y a des phrases avec 24 trous, qu'il faut remplir avec l'un des verbes 
imposés : ligga, stå ou sitta. Les phrases incluent suffisamment de contexte pour rendre la situation spatiale claire, par exemple :

\section{statistique à
étude pilote.}

nous voyons l'évolution des apprenants entre les trois tests. Avant de recevoir l'enseignement spécialisé, ils ont produit $69,8 \%$ de bons choix, et pour les deux tests suivants, passés trois puis douze semaines après, les résultats atteignent presque $90 \%$ (89,5\% et $89,8 \%$ respectivement). Il semblerait donc que les explications ciblées sur la sémantique des verbes ont été fructueuses.

Nordiques, 39 | 2020 
Figure 1 : Proportion de choix corrects pour les trois tests.

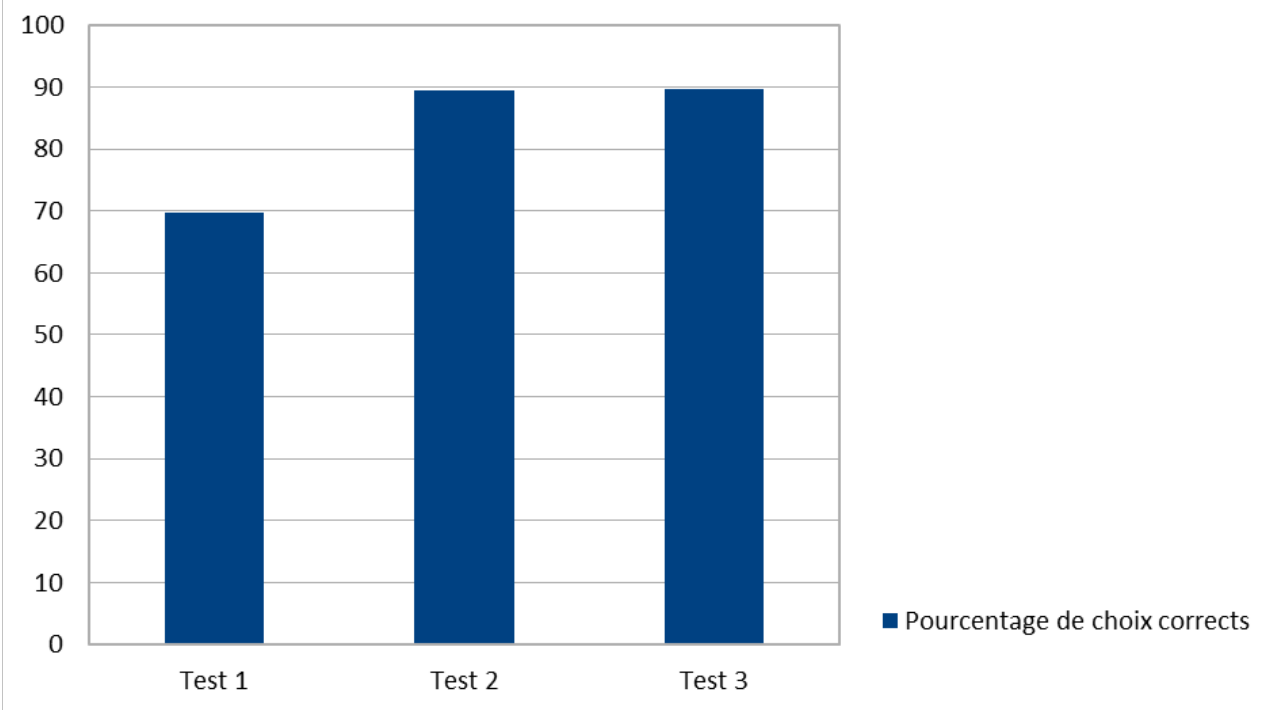

Si l'on se penche sur les usages corrects par verbe d'une façon plus qualitative, on voit dans la figure (2) ci-dessous que la tendance à faire le bon choix augmente de manière générale. En effet, ayant choisi seulement $52,74 \%$ du verbe sitta d'une façon correcte au premier test, les étudiants l'utilisent dans $90,47 \%$ des cas possibles au deuxième test, avec une légère baisse pour le dernier test $(85,71 \%)$. Pour le verbe stå, on voit une augmentation constante de l'usage correct, avec $70,32 \%$ au premier test, $95,23 \%$ au deuxième et $97,95 \%$ au troisième test. Il n'y a que le verbe ligga qui souffre d'une baisse d'usage au deuxième test, ayant un usage de $81,53 \%$ au premier test, $76,66 \%$ au deuxième, mais une augmentation importante $87,14 \%$ au troisième.

Figure 2 : Utilisations correctes des verbes de position par test

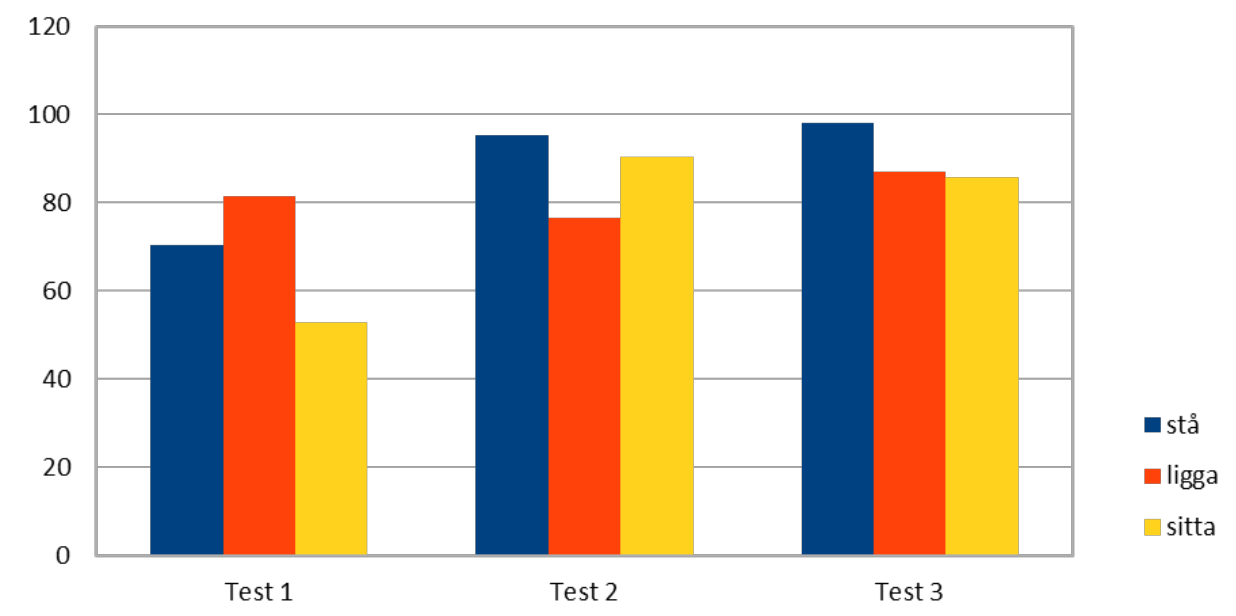

Il est également intéressant d'examiner les usages erronés, afin de distinguer une prédisposition pour un des verbes chez les apprenants. Selon la figure (3) ci-dessous, il semblerait que ce soit le verbe stå qui jouit de cette préférence, car c'est le verbe qui est utilisé le plus dans les situations qui ne le requièrent pas. 
Figure 3 : Choix erroné de verbe de position par test, par pourcentage

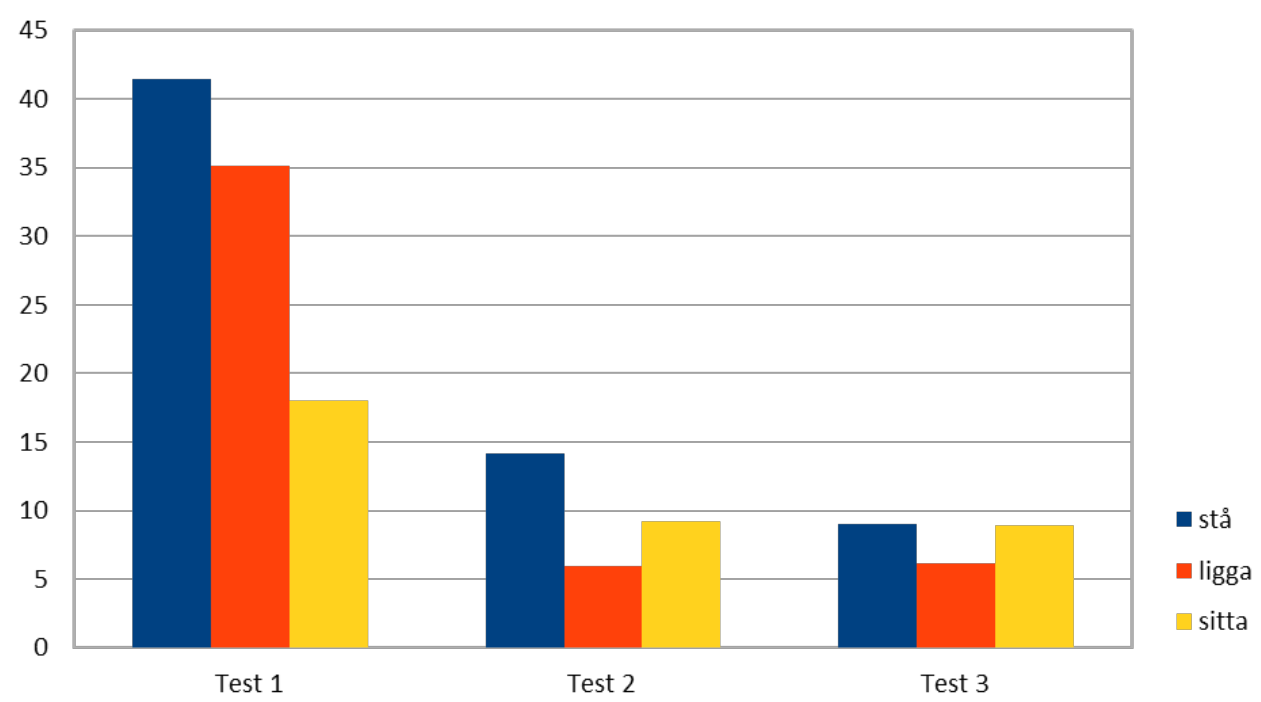

Pour les deuxième et troisième tests, c'est toujours stå qui est le plus utilisé d'une façon erronée, tandis qu'un mauvais choix du verbe ligga se fait plus rare. Le verbe sitta souffre d'une plus grande sur-utilisation que ligga. Nous allons revenir sur les raisons éventuelles de ces phénomènes dans la partie consacrée à l'analyse.

Pour les contextes requérant le verbe stå, aucune situation n'a recueilli $100 \%$ de bonnes réponses dès le premier test, mais quatre situations l'ont fait au deuxième tour, à savoir pour une personne restant devant la porte, une personne faisant la queue, les assiettes dans le placard, et le lait dans le réfrigérateur. Pour cette dernière situation, une personne sur 14 s'est trompée au dernier test, en encodant le lait par le verbe sitta, tandis que les autres situations restent avec $100 \%$ de bonnes réponses à la fin. La situation spatiale contenant des assiettes posées dans un placard a recueilli seulement trois choix de stå sur 13 personnes au premier test, mais $100 \%$ aux deux tests suivants. Les situations de la voiture dans le garage et du lit dans une chambre, devant être encodés par stå, ont inspiré 3 respectivement 4 personnes à utiliser le verbe ligga au premier test. Même au deuxième test, les apprenants ne répondent pas par le verbe stå à $100 \%$ pour ces situations, mais une personne sur 12 insiste sur l'usage de ligga pour la voiture, et deux apprenants utilisent ce verbe pour encoder le lit.

Pour deux situations spatiales requérant ligga, les apprenants avaient un taux de réussite de $100 \%$ tout au long des tests, à savoir celles décrivant un journal sur une table et une relation géotopographique respectivement. Deux autres situations de ligga avaient des taux de réponses correctes très élevés et ont atteint les $100 \%$ dès le deuxième test. Il s'agit des clés ainsi que du stylo sur la table. Pour l'araignée morte, elle fut encodée correctement avec ligga au dernier test, tandis que pour les deux précédents, deux personnes par test ont choisi trois fois står et une fois sitter.

La situation spatiale avec la moins bonne évolution entre les tests tous verbes confondus concerne le ballon sur la pelouse. En effet, cet objet symétrique doit être encodé par le verbe ligga, mais seulement 38,4\% l'ont fait au premier test, $41,6 \%$ pour le deuxième questionnaire, et seulement $57,1 \%$ des apprenants ont intégré cet usage au dernier test. 

représentations mentales de la sémantique des verbes, a aidé les apprenants dans leur acquisition, car l'usage correct des verbes augmente dans le temps. Mais l'on peut voir que la variation sémasiologique des verbes pose un souci, soit le fait que le verbe peut être utilisé dans un grand nombre de situations, qui n'ont pas l'air d'avoir quelque chose en commun, est difficile à intégrer par les apprenants.

41 Selon les résultats présentés ci-dessus, il semblerait que stå est le verbe le plus facilement choisi ${ }^{34}$, même pour les situations où ses paramètres ne s'appliquent pas, que ce soit avant ou après l'enseignement spécialisé. Il est possible que cela soit dû au fait que ce verbe est le plus fréquent des trois verbes de position. Une autre explication est envisageable: la sur-utilisation de ce verbe semble dépendre de la situation de l'oiseau, qui doit être encodé par sitta, mais qu'une grande partie des apprenants a décrit par le verbe stå, très probablement, et très logiquement, en pensant aux pieds de 
l'animal, sur lesquels reposent celui-ci. Dans la même logique, la personne criminelle se trouvant en prison fut encodée par stå 7 fois sur 13 au premier test, et l'usage de ce verbe persiste au deuxième test ( 3 sur 12) ainsi qu'au dernier test (4 sur 14). Il s'agit très probablement de l'image canonique que nous avons de l'être humain en position debout qui a induit les apprenants à choisir ce verbe. Or, pour une personne en prison, c'est le paramètre de l'inclusion qui prend le dessus, en combinaison avec la position fréquente d'une personne inactive, à savoir la position assise.

Dans la plupart des études précédentes, le concept d'horizontalité était le plus fréquemment exprimé par les apprenants L2, même lorsqu'il n'était pas correct. Comme nous pouvons le voir, tel n'est pas le cas dans cette étude-ci, mais les apprenants se sont surtout approprié le verbe stå. Malgré cela, l'on peut constater que ligga est choisi lorsqu'une horizontalité est existante (mais pas actualisée dans la situation spatiale actuelle) comme pour les assiettes et le lit, mais aussi pour le tableau et le poster, qui ont une extension plate. Cela montre une certaine compréhension préalable de la sémantique basique du verbe, qui encode un être humain en position allongée avec une extension horizontale. Toutefois, selon la figure (3) ci-dessus, illustrant le choix erroné des verbes, on observe que ligga est choisi dans $35,1 \%$ des cas pendant le premier test. Il est possible d'expliquer cette sur-utilisation par une généralisation du concept géotopographique par les apprenants à toute sorte de localisation. En effet, cet usage de ligga est le premier appris lorsqu'on fait des études de suédois langue étrangère. Pour le deuxième test, les apprenants avaient donc intégré qu'une certaine analyse de la situation spatiale était requise afin de faire le choix correct. Une sorte d'évitement de ligga, de peur de le sur-utiliser, peut éventuellement être l'explication de la chute d'usage de ce verbe, car seulement 5,9\% des choix erronés concernent ligga au deuxième test. Une règle claire et constante pour l'usage de ce verbe concerne les objets ronds et symétriques, où seul le verbe ligga peut être utilisé, mais cette règle n'a pas été intégrée par les apprenants. Il faudra donc insister davantage sur ce point pendant l'enseignement de ces verbes dans le futur.

Selon nos études antérieures ${ }^{35}$, le verbe sitta est acquis en dernier, d'une part probablement en raison de l'usage moins fréquent du verbe dans la langue quotidienne, et d'autre part en raison des paramètres plus sémantiquement complexes, à savoir le contact et l'inclusion. Ces résultats se confirment également pour cette étude, car c'est le verbe le moins utilisé au premier test, et même après les explications détaillées, il existe une certaine hésitation, voire une sur-interprétation des paramètres, par exemple dans les cas de la voiture dans le fossé et des couverts dans le tiroir. Pour la voiture, une personne sur $13(7,7 \%)$ a choisi sitta au premier test, mais au deuxième test, 4 sur $12(33,3 \%)$ l'ont préféré, tandis qu'au troisième test, cette tendance baisse avec 2 sur 14 (14,3\%). Pour les couverts, la tendance s'inverse, car aucun apprenant n'opte pour sitta au deuxième test, mais l'usage revient au troisième test, où 2 personnes sur 14 (14,3\%) le choisissent.

Dans la méthode employée pour notre étude, nous avons imposé les trois verbes de position, et ainsi nous avons montré aux apprenants la contrainte de l'encodage de ces verbes pour les situations d'emplacement. Malgré cela, un apprenant a écrit är « est » une fois au premier test, et au dernier test, il y a eu trois ellipses verbales. Cela montre qu'en dépit d'une consigne imposée et limitée, un locuteur francophone peut préférer une expression neutre à une expression positionnelle, ce qui reflète les résultats de notre étude ${ }^{36}$. Ainsi, la différence typologique entre les deux langues rajoute une 
difficulté pour nos étudiants dans l'apprentissage des verbes de localisation suédois, puisque, comme précédemment observé, le français n'exprime pas, ou très rarement, l'orientation de l'objet dans une situation d'emplacement.

\section{Conclusion}

Cette étude pilote n'était qu'un premier pas dans la recherche d'une méthode pour enseigner les verbes de position suédois. Les limitations de l'étude sont nombreuses, notamment la taille du groupe d'informateurs, qui ne nous permet pas de faire de la statistique. En outre, la méthode du questionnaire à choix multiple impose les verbes de position, et ne laisse pas la possibilité à l'apprenant d'utiliser un verbe neutre qui lui paraît sans doute plus naturel, au vu des résultats dans Hellerstedt ${ }^{37}$. Dans le futur, les résultats d'un groupe de contrôle, passant les tests sans avoir reçu l'enseignement ciblé, seront inclus dans l'étude.

Le choix de méthode d'enseignement semble en revanche prometteur, et nous continuerons ces explications basées sur la sémantique cognitive dans le futur, en élaborant les explications comme proposé dans cet article. Toutefois, une plus grande attention sera donnée au trait classificateur des verbes de position, selon lequel ceux-ci semblent catégoriser les noms. En outre, pour certaines situations, il faudra relever l'interaction entre la Figure et le Fond, qui parfois joue un grand rôle dans le choix de verbe.

\section{NOTES}

1. Le verbe hänga « être suspendu » n'est en général pas inclus dans ce groupe. Une explication à avancer est peut-être qu'il n'encode pas une posture humaine canonique.

2. Maria Hellerstedt, Localiser et/ou positionner. Une étude sur la façon dont on exprime l'emplacement des personnes et des objets en suédois, Mémoire de M2 (non publié en linguistique, Université de Lille 3, 28 juin 2005, 10 volumes, 117 pages, mention Très bien.

3. Voir Maria Hellerstedt, L'utilisation et l'acquisition des verbes de position en suédois L1 et L2, Thèse de doctorat non-publiée en linguistique, Université de Paris Sorbonne, 29 novembre 2013, 15 volumes, 501 pages, mention Très honorable avec les félicitations du jury. Dans ce travail est présentée une étude sur l'acquisition des verbes de position par les enfants suédophones âgés de 4,7 et 10 ans.

4. Leonard Talmy, Toward a Cognitive Semantics, vol II, Cambridge MA, MIT Press, p. 25.

5. Irène Baron \& Michael Herslund, «Langues endocentriques et langues exocentriques. Approche typologique du danois, du français et de l'anglais ", Langue française, $\mathrm{n}^{\circ} 145,2005$, p. 35-53.

6. Irène Baron \& Michael Herslund, op. cit. p. 36.

7. Michael Herslund, "Når ordene ikke svarer til hinanden. Sprogtypologi og oversættelse », Sprogforum, $\mathrm{n}^{\circ} 47$, p. 39.

8. Maarten Lemmens \& Julien Perrez, « On the use of posture-verbs by French-speaking learners of Dutch : a corpus-based study ", Cognitive Linguistics, n²1-2, 2010, p. 325. 
9. Dans cet article, nous ne traiterons que des usages concrets, à savoir la localisation d'une personne ou d'un objet dans un endroit concret.

10. Voir la thèse de Ulrika Kvist Darnell, Pseudosamordningar i svenska, särskilt sådana med sitta, ligga och stå, Stockholm, Stockholms universitet, 2008, pour une description détaillée sur les constructions progressives en suédois. Un exemple : Hon sitter och läser « Elle est en train de lire ». 11. Åke Viberg, "Towards a lexical profile of the Swedish verb lexicon», Sprachtypologie und Universalienforschung, $\mathrm{n}^{\circ}$ 59(1), 2006, p. 108.

12. Cf Åke Viberg, " Posture verbs. A multilingual study ». Languages in Contrast, 13:2, 2013, p. 145.

13. Cf Ulrika Jakobsson, « Familjelika betydelser hos stå, sitta och ligga. En analys ur den kognitiva semantikens perspektiv ». Nordlund 21. Småskrifter från institutionen för nordiska språk $i$ Lund, 1996, p. 1-66; Maarten Lemmens, "Caused posture: Experiential patterns emerging from corpus research ». In Stefanowitch, Anatol \& Gries, Stefan (éds), Corpora in Cognitive Linguistics, Vol. II: The Syntax_Lexis Interface, Amsterdam/Philadelphia, John Benjamins, 2006, p. 261-298; Maarten Lemmens \& Julien Perrez, op. cit., 2010.; Maria Hellerstedt, op. cit., 2013.

14. Cf Leonard Talmy, op. cit., 2000 ; Mark Johnson, The body in the mind - the bodily basis of meaning, imagination and reason, Chicago, University of Chicago Press, 1987.

15. Inspirée par Maarten Lemmens, op. cit., 2006, et Maarten Lemmens \& Julien Perrez, op. cit., 2010.

16. Ibid.

17. $C f$ Maarten Lemmens, "Tracing referent location in oral picture descriptions ", in Wilson, Andrew, Rayson, Paul et McEnery, Tony, A Rainbow of Corpora - Corpus Linguistics and the Languages of the World, Munich, Lincom-Europa, 2002a, p. 73-85.

18. Les paramètres décrits ici sont surtout applicables aux usages spatiaux des verbes de position. Pour une discussion des images schématiques derrière les usages métaphoriques, voir Ulrika Jakobsson, op. cit. 1996.

19. Cf Åke Viberg, « Lexikal andraspråksinlärning. Hur polsk-, spansk- och finskspråkiga lär in svenskans placerarverb ", in SUM-rapport 2. Stockholm, Stockholm university, 1985, p. 5-91; Åke Viberg, "Basic verbs in lexical progression and regression", in Burmeister, Petra, Piske, Thorsten \& Rohde, Andreas (éds), An Integrated View of Language Development. Papers in Honor of Henning Wode, Trier, Wissenschaftlicher Verlag, 2002, p. 109-129; Maria Hellerstedt, "L'acquisition de la distribution sémantique dans une L2. Evénements de placement : l'exemple du suédois ", in Trévisiol-Okamura, Pascale \& Gobet, Stéphanie (éds), Acquisition des langues. Approches comparatives et regards didactiques, Rennes, Presses Universitaires de Rennes, 2017, p. 63-92.

20. Marianne Gullberg, "Reconstructing verb meaning in a second language. How Englishspeakers of L2 Dutch talk and gesture about placement ", Annual Review of Cognitive Linguistics, $\mathrm{n}^{\circ}$ 7, 2009, p. 221-244.

21. Teresa Cadierno, Iraide Ibarretxe-Antunano \& Alberto Hijazo-Gascón, «Semantic categorization of placement verbs in L1 and L2 Danish and Spanish ", Language learning, n 66-1, 2016, p. 191-223.

22. Rod Ellis, The Study of Second Language Acquisition, Oxford, Oxford University Press, 1994.

23. Maria Hellerstedt, op. cit., 2013.

24. Cf. Maarten Lemmens, "The semantic network of Dutch zitten, staan and liggen", in Newman, John (éd), The Linguistics of Sitting, Standing, and Lying, Amsterdam \& Philadelphia, John Benjamins, 2002b, p. 103-140 ; Maarten Lemmens \& Julien Perrez, op. cit., 2010 ; Julien Perrez \& Maarten Lemmens, "A quantitative analysis of the use of posture verbs by French-speaking learners of Dutch ", Cognitextes, n 8 (mis en ligne le 28 décembre 2012, consulté le 9 septembre 2019) URL : http://cognitextes.revues.org/609.

25. Maarten Lemmens \& Julien Perrez, op. cit., 2010 ; Julien Perrez \& Maarten Lemmens, op. cit., 2012. 
26. Maria Hellerstedt, «L'acquisition de l'expression de l'emplacement en suédois L1 et L2 », in Estève, I et al., Autour des langues et du langage - Perspective pluridisciplinaire, Grenoble, PUG, 2011, p. 37-44 ; Maria Hellerstedt, op. cit., 2013 ; Maria Hellerstedt, « När fransmän ska lära sig svenska positionsverb", in Reuter, Hedwig (éd), Le nord en français : traduction, interprétation, interculturalité, Université de Mons, C.I.P.A, 2014, p. 87-100 ; Maria Hellerstedt, op. cit., 2017.

27. Maarten Lemmens \& Julien Perrez, op. cit., 2010, p. 329.

28. Ibid., p. 344.

29. Cf. Åke Viberg, op. cit., 1985 ; Maarten Lemmens, op. cit., $2002 \mathrm{~b}$.

30. Maria Hellerstedt, op. cit., 2013, p. 414.

31. Ibid., p. 174.

32. CECRL, Cadre européen commun de référence, Conseil de l'Europe, 2000, URL: http:// www.coe.int/T/DG4/Linguistic/Source/Framework_FR.pdf

33. Maria Hellerstedt, op. cit., 2013, 2014.

34. Cf Maarten Lemmens \& Julien Perrez, op. cit., 2010.

35. Maria Hellerstedt, op. cit., 2011, 2013, 2014, 2017.

36. Ibid.

37. Ibid.

\section{RÉSUMÉS}

Les verbes de position suédois posent des problèmes aux apprenants francophones de suédois langue étrangère, en raison du manque de différenciation sémantique dans la langue française. Dans cet article, nous évaluons une méthode d'enseignement de ces verbes qui repose sur la sémantique cognitive et ses schémas d'image. Les résultats montrent une tendance à la compréhension de ceux-ci par les étudiants de suédois d'une université française, ainsi qu'un apprentissage rapide des verbes de position. Malgré cela, il reste des hésitations et des erreurs chez les apprenants, certainement dues à la différence typologique entre les deux langues ainsi qu'à la complexité sémantique des verbes en question.

The Swedish posture verbs are difficult to learn by French-speaking learners of Swedish as a second language. This is due to the lack of such semantic differentiation in French when it comes to this kind of verbs. In this article we evaluate a teaching method leaning on cognitive semantics and image schemata. The results show that the students learning Swedish at a French university have a tendency to understand these image schemata, and they learn the posture verbs quickly. In spite of this, they still hesitate and make mistakes, which are probably due to the typological differences betweend these two languages, as well as to the semantical complexity lying in these verbs.

INDEX

Mots-clés : enseignement, apprentissage des langues, langue suèdoise, sémantique

Keywords : language learning, Swedish, semantic 


\section{AUTEUR}

\section{MARIA HELLERSTEDT}

Maria Hellerstedt est maître de conférences de suédois au Département de Langues Etrangères appliquées à l'université de Lille. 